Introduction Supplemental oxygen has long been used as a therapeutic agent in the management of ischaemic chest pain, but without a sound scientific basis. We proposed to investigate the effect of high-flow oxygen on coronary physiology, invasively and non-invasively, in those with severe coronary artery disease (CAD).

Methods 30 volunteers and 30 patients with severe CAD were allocated to receive high-flow air and oxygen in a random blinded order. They underwent cardiovascular magnetic resonance (CMR) T2* blood-oxygen level dependent (BOLD) and dynamic contrast-enhanced perfusion imaging. Myocardial blood flow (MBF), perfusion reserve (MPR), first-pass extraction fraction $(\mathrm{E})$, blood volume fraction $\left(\mathrm{v}_{\mathrm{b}}\right)$ and capillary permeability-surface area product (PS) were quantified using bookend $\mathrm{T}_{1}$ based non-linearity correction and distributed parameter model constrained deconvolution.

Invasive coronary physiology measurements were performed in severe CAD patients (Fractional Flow Reserve <0.75), also randomised to high-flow air and oxygen, using a pressure wire (Radi, St Jude Medical, Uppsala, Sweden). The mean transit time of a room temperature $3-4 \mathrm{ml}$ bolus of saline was recorded at rest and hyperaemic (adenosine) stress for both inspired gases. Resistance Index (RI), Resistance Reserve Index (RRI), Index of Microvascular Resistance (IMR) and Coronary Flow Reserve (CFR) were derived.

Results In volunteers the change in BOLD Air-T2* was $6.1 \pm$ $7.5 \mathrm{~ms}$ vs. $\mathrm{O}_{2}-\mathrm{T} 2 * 7.8 \pm 8.1, \mathrm{P}=0.575$. Rest Air-MBF was $0.88 \pm 0.40 \mathrm{ml} / \mathrm{min} / \mathrm{g}$ vs. $\mathrm{O}_{2}-\mathrm{MBF} 0.95 \pm 0.45, \mathrm{P}=0.168$ and hyperaemic Air-MBF $3.46 \pm 1.67$ vs. $\mathrm{O}_{2}-\mathrm{MBF} 3.35 \pm$ 2.05, $\mathrm{P}=0.821$.

In patients, ischaemic region change in BOLD Air-T2* was $1.1 \pm 6.6 \mathrm{~ms}$ vs. $\mathrm{O}_{2}-\mathrm{T} 2 * 2.7 \pm 8.2 \mathrm{~ms}, \mathrm{P}=0.583$ compared to Air-T2* $11.2 \pm 8.7$ vs. $\mathrm{O}_{2}-\mathrm{T} 2 * 2.9 \pm 2.5, \mathrm{P}=0.004$ in remote myocardium. Remote Air-MBF was $0.81 \pm 0.19$ vs. $\mathrm{O}_{2}-\mathrm{MBF} 0.76 \pm 0.15, \mathrm{P}=0.087$ and hyperaemic Air-MBF $1.31 \pm 0.047$ vs. $\mathrm{O}_{2}-\mathrm{MBF} 1.50 \pm 0.53, \mathrm{P}=0.249$; whilst ischaemic region Air-MBF was $0.83 \pm 0.20$ vs. $\mathrm{O}_{2}-\mathrm{MBF} 0.85$ $\pm 0.28, \mathrm{P}=0.725$ and hyperaemic Air-MBF $1.26 \pm 0.055$ vs. $\mathrm{O}_{2}-\mathrm{MBF} 1.35 \pm 0.59, \mathrm{P}=0.407$. Rest ischaemic Air-E was $0.69 \pm 0.14$ vs. $\mathrm{O}_{2}-\mathrm{E} 0.66 \pm 0.17, \mathrm{P}=0.408$ and remote Air-E was $0.73 \pm 0.13$ vs. $\mathrm{O}_{2}-\mathrm{E} 0.64 \pm 0.15, \mathrm{P}=$ 0.044 . Ischaemic region Air-PS was $0.67 \pm 0.38 \mathrm{ml} / \mathrm{min} / \mathrm{g}$ vs. $\mathrm{O}_{2}$-PS $0.58 \pm 0.32, \mathrm{P}=0.227$; whilst remote region Air-PS was $0.72 \pm 0.38$ vs. $\mathrm{O}_{2}$-PS $0.53 \pm 0.32, \mathrm{P}=0.024$.

Invasively there was an increase in rest transit time $0.69 \pm$ 0.35 vs. $0.97 \pm 0.50, \mathrm{P}=0.001$, resistance index $51.0 \pm$ 26.6 vs. $75.0 \pm 38.0, \mathrm{P}<0.001$, coronary flow reserve 2.21 \pm 1.30 vs. $2.90 \pm 1.51, \mathrm{P}=0.019$ and resistance reserve index $2.62 \pm 1.37$ vs. $3.54 \pm 1.74, \mathrm{P}=0.006$ in the air vs oxygen groups respectively.

Conclusion Supplemental high-flow oxygen (and hence hyperoxaemia) results in increased microvascular resistance in patients with severe $\mathrm{CAD}$, demonstrated during invasive coronary physiology studies. We have also shown with novel CMR techniques that, in patients with severe CAD, whilst there is no difference in absolute myocardial blood flow, high-flow supplemental oxygen results in a reduction in first-pass extraction fraction and capillary permeability-surface area product. This results in a reduction in myocardial oxygenation in the remote, non-ischaemic, myocardium. Supplemental high-flow oxygen should therefore be avoided in patients who are not hypoxic.

\section{E MICRORNA-140-5P AND SMURF1 REGULATE PULMONARY ARTERIAL HYPERTENSION}

\begin{abstract}
1,2Alexander MK Rothman*, 'Nadine D Arnold, 'Josephine A Pickworth, 'James Iremonger, ${ }^{3}$ Loredana Ciuclan, ${ }^{3}$ Robert Allen, ${ }^{4}$ Sabine Guth-Gundel, ${ }^{5}$ Mark Southwood, ${ }^{5}$ Nicholas W Morrell, ${ }^{6}$ Matthew Thomas, ${ }^{1}$ Sheila E Francis, ${ }^{3}$ David J Rowlands, ${ }^{1}$ Allan Lawrie. ${ }^{1}$ Department of Cardiovascular Science, University of Sheffield, Sheffield, UK; ${ }^{2}$ Sheffield Teaching Hospitals NHS Trust, Sheffield, UK; ${ }^{3}$ Novartis Institutes for Biomedical Research, Cambridge, MA, USA; ${ }^{4}$ Novartis Institutes for Biomedical Research, Basel, Switzerland; ${ }^{5}$ Department of Medicine, University of Cambridge School of Clinical Medicine, Addenbrooke's and Papworth Hospitals, Cambridge, UK; ${ }^{6}$ AstraZeneca R\&D, Respiratory, Inflammation and Autoimmunity (RIA) Innovative Medicines, Building AC461, SE-431 83 Mölndal, Sweden; *Presenting Author
\end{abstract}

\subsection{6/heartjil-2016-309890.224}

Background Clinical therapies for the treatment of pulmonary arterial hypertension (PAH) target vasoconstriction. However, the proliferative pulmonary vascular remodelling that drives disease persists contributing to significant patient morbidity and mortality. MicroRNA (miR) are short non-coding RNA that mediate post-transcriptional regulation of mRNA targets. We hypothesise that dysregulation of miR leads to de-repression of cellular targets central to disease pathogenesis.

Objective To identify dysregulated circulating $\mathrm{miR}$ in patients with $\mathrm{PAH}$, determine their phenotypic effect using in vitro and in vivo models and identify key mechanistic regulators that may represent novel therapeutic targets.

Methods and results Expression of miR-140-5p was reduced in whole blood samples from patients with PAH. Reduced expression of miR-140-5p at the time of diagnosis identified patients with adverse clinical characteristics. Transfection with miR-140-5p inhibitor resulted in increased proliferation PASMC and de-repression of key targets. Nebulised delivery of miR-140-5p mimic attenuated progression of established $\mathrm{PAH}$ in the SuHx rat model. Network and pathway analysis identified SMAD Specific E3 Ubiquitin Protein Ligase 1 (SMURF1) as a key miR-140-5p target. Whole blood mRNA and pulmonary vascular immunoreactivity of SMURF1 was increased in patients with PAH implicating SMURF1 in the pathology of human disease. Direct regulation of SMURF1 by miR-140-5p was demonstrated in-vitro by 3'UTR luciferase activity. Both miR-140-5p mimic and SMURF1 siRNA increased BMP response element activity identifying SMURF1 as a key negative regulator of BMP signalling in PASMC. Genetic ablation of SMURF1 in C57BL6 mice conferred allele dependent protection from SuHx induced PAH.

Conclusions These studies suggest that miR-140 and SMURF1 are key regulators of BMP signalling and disease pathology in $\mathrm{PAH}$ and highlight SMURF1 as a potential novel therapeutic target. 